\title{
How Much Sleep Does an Elite Athlete Need?
}

\author{
Charli Sargent, Michele Lastella, Shona L. Halson, and Gregory D. Roach
}

\begin{abstract}
Purpose: Anecdotal reports indicate that many elite athletes are dissatisfied with their sleep, but little is known about their actual sleep requirements. Therefore, the aim of this study was to compare the self-assessed sleep need of elite athletes with an objective measure of their habitual sleep duration. Methods: Participants were 175 elite athletes $(\mathrm{n}=30$ females), age 22.2 (3.8) years (mean [SD]) from 12 individual and team sports. The athletes answered the question "how many hours of sleep do you need to feel rested?" and they kept a self-report sleep diary and wore a wrist activity monitor for $\sim 12$ nights during a normal phase of training. For each athlete, a sleep deficit index was calculated by subtracting their average sleep duration from their self-assessed sleep need. Results: The athletes needed $8.3(0.9)$ hours of sleep to feel rested, their average sleep duration was $6.7(0.8)$ hours, and they had a sleep deficit index of 96.0 (60.6) minutes. Only $3 \%$ of athletes obtained enough sleep to satisfy their self-assessed sleep need, and $71 \%$ of athletes fell short by an hour or more. Specifically, habitual sleep duration was shorter in athletes from individual sports than in athletes from team sports $\left(F_{1,173}=13.1, P<.001 ; d=0.6\right.$, medium), despite their similar sleep need $\left(F_{1,173}=1.40, P=.24 ; d=0.2\right.$, small). Conclusions: The majority of elite athletes obtain substantially less than their self-assessed sleep need. This is a critical finding, given that insufficient sleep may compromise an athlete's capacity to train effectively and/or compete optimally.
\end{abstract}

Keywords: sleep duration, sleep need, sleep deficit, recovery

The true function of sleep is not yet fully understood, but it plays an important role in energy conservation, ${ }^{1}$ nervous system recuperation, ${ }^{2}$ host-defense mechanisms,${ }^{3}$ and restoration of optimal performance ${ }^{4}$-all of which are critical for elite athletes. The amount of sleep required to maintain these functions is a natural and relevant question, and many athletes and coaches seek guidance regarding targets for sufficient sleep duration.

The appropriate sleep duration recommended by the US National Sleep Foundation is 7 to 9 hours for young adults (18$25 \mathrm{y})$ and 7 to 8 hours for other adults (26-64 y). ${ }^{5}$ These recommendations were developed by an 18-member expert panel and are based on a systematic review of medical and scientific research regarding the consequences of either too little, or too much, sleep for health and performance. When compared against these general benchmarks, elite athletes typically obtain less sleep than is recommended $^{6-11}$ or the minimum amount of sleep that is recommended. ${ }^{12-14}$

The National Sleep Foundation's guidelines are useful for identifying potential deficiencies in habitual sleep duration at a broad level, but they are not sensitive to individual differences in sleep need. In general, many aspects of mental performance are impaired by sleep loss in a dose-dependent fashion - that is, the less sleep obtained, the poorer the performance. ${ }^{15,16}$ However, there is considerable variability in the individual response to sleep loss-some maintain good levels of performance, while others perform poorly. ${ }^{17}$ At present, we do not have a good understanding of how much sleep an elite athlete needs, nor do we know whether they obtain their required sleep need on a habitual basis. It is possible that some athletes may require less

Sargent, Lastella, and Roach are with the Appleton Institute for Behavioural Science, Central Queensland University, Adelaide, SA, Australia. Halson is with the School of Behavioural and Health Sciences, Australian Catholic University, Brisbane, QLD, Australia. Sargent (charli.sargent@cqu.edu.au) is corresponding author. sleep than recommended by the National Sleep Foundation, while others may require more.

The aims of the present study were to (1) identify the subjective sleep need of elite athletes and compare it with an objective measure of their habitual sleep duration; (2) examine the relationships between habitual sleep onset, habitual sleep offset, and habitual sleep duration; (3) compare sleep variables between individual and team sports; and (4) compare sleep variables between sexes. We hypothesize that objective habitual sleep duration in elite athletes will be lower than their subjective sleep need.

\section{Methods}

\section{Participants}

A total of 175 elite athletes from 12 sports (Australian Rules football, basketball, cricket, kayaking, mountain biking, race walking, road cycling, rugby union, soccer, swimming, track cycling, and triathlon) gave informed consent to participate in the study (Table 1). Athletes were volunteers from national teams of which the coaching staff had expressed an interest in having the sleep of their athletes monitored. Participants were excluded if they were training or sleeping at altitude, if they were injured, if they reported a clinical diagnosis of a sleep disorder, or if they had undertaken transmeridian travel in the 2 weeks prior to data collection. According to the National Sleep Foundation's Guidelines, ${ }^{5} 11$ athletes were categorized as teenagers, 128 were classified as young adults, and 26 were classified as adults. The study was approved by the Human Research Ethics Committees of Central Queensland University and the University of South Australia.

\section{Procedures}

Athletes' sleep/wake behavior was monitored for a minimum of 4 nights during a normal phase of training outside of competition using self-report paper sleep diaries in conjunction with wrist 
Table 1 Participant Characteristics

\begin{tabular}{|c|c|c|c|}
\hline Participants & $\mathbf{n}$ & Age, y & BMI, $\mathrm{kg} / \mathrm{m}^{2}$ \\
\hline Total & 175 & $22.2(3.8)$ & $24.3(3.7)$ \\
\hline Men & 145 & $22.4(3.7)$ & $24.8(3.8)$ \\
\hline Women & 30 & $21.1(4.5)$ & $21.8(2.2)$ \\
\hline \multicolumn{4}{|l|}{ Sport } \\
\hline Alpine skiing & 1 & 22.0 (NA) & NA (NA) \\
\hline Australian Rules football & 43 & $22.3(3.3)$ & $24.3(3.9)$ \\
\hline Basketball & 11 & $17.3(0.9)$ & $23.2(1.4)$ \\
\hline Cricket & 17 & $23.9(3.8)$ & $24.4(1.1)$ \\
\hline Diving & 1 & 18.0 (NA) & 23.7 (NA) \\
\hline Kayaking & 2 & $24.0(0.0)$ & $26.5(2.5)$ \\
\hline Mountain biking & 7 & $25.7(4.7)$ & $20.9(1.0)$ \\
\hline Race walking & 4 & $22.5(4.1)$ & $20.3(1.4)$ \\
\hline Road cycling & 9 & $19.2(1.2)$ & $22.3(1.4)$ \\
\hline Rugby union & 29 & $24.6(3.5)$ & $29.8(2.9)$ \\
\hline Soccer & 20 & $20.3(3.5)$ & $23.8(1.7)$ \\
\hline Swimming & 8 & $22.6(4.9)$ & $22.8(2.0)$ \\
\hline Track cycling & 6 & $23.3(2.0)$ & $26.3(1.7)$ \\
\hline Triathlon & 17 & $21.2(2.8)$ & $20.4(1.5)$ \\
\hline
\end{tabular}

Abbreviations: BMI, body mass index; NA, not applicable. Note: Data are presented as mean (SD).

activity monitors. Each athlete wore an activity monitor on the same wrist throughout the data collection period, except when showering, swimming, or training. The sleep diaries were used to record 2 pieces of information for each nighttime sleep: start date/ time and end date/time. Daytime naps were not recorded. Athletes were instructed to complete their sleep diary each morning 30 minutes after waking. There was no experimental manipulation of the athletes' training schedules or sleep/wake behaviors, and the athletes were free to consume training supplements, caffeine, or alcohol during the data collection period. Information regarding medication use (including sleeping pills) was not collected. Prior to the commencement of data collection, athletes completed a series of questions presented in the front of the sleep diary to assess sleep need, sleep satisfaction, and sleep quality. Data collected from some of the athletes included in the present study have been reported elsewhere. ${ }^{6-9,18,19}$

\section{Subjective and Objective Sleep Measures}

A series of pen/paper questions were used to capture information regarding athletes' perspectives of their sleep. These included:

1. Sleep need (in hours), assessed with the question "How many hours of sleep do you need to feel rested?";

2. Sleep satisfaction (in arbitrary units), assessed with the question "How satisfied are you with the amount of sleep you get?"; responses were rated using a 10-point scale, where $1=$ very dissatisfied and $10=$ very satisfied;

3. Sleep quality (in arbitrary units), assessed with the question "Overall, how would you rate the quality of your sleep?"; responses were rated using a 6-point Likert scale, where 1 =very poor, 2 =poor, $3=$ fair, $4=$ good, $5=$ very good, and $6=$ excellent.

Due to availability, 2 different models of activity monitorproduced by a sole manufacturer-were used in this study
(Actiwatch-64 and Actical Z-series; Philips Respironics, Bend, OR). The monitors were configured to sum and store data in 1-minute epochs based on activity counts from a piezoelectric accelerometer with a sensitivity of $0.05 \mathrm{~g}$ and a sampling rate of $32 \mathrm{~Hz}$. Data from the sleep diary and activity monitor were used to determine when participants were awake and when they were asleep. Essentially, all time was scored as wake unless: (1) the sleep diary indicated that the athlete was lying down attempting to sleep and (2) the activity counts from the monitor were sufficiently low to indicate that the athlete was immobile. ${ }^{20}$ When these 2 conditions were satisfied simultaneously, time was scored as sleep. In this study, sensitivity was set at medium, which corresponds to a threshold activity count of 40. (Please note: Sensitivity can be set at "low" for elite athletes instead of medium, but this may only be suitable when using the Actiwatch-64.) This scoring process was conducted using a Philips Respironics' Actiwatch algorithm. Validation studies comparing wrist activity monitors with polysomnography report high levels of agreement in healthy adults $(88 \%)^{21}$ and well-trained athletes $(81 \%-90 \%) .^{22}$

For each athlete, the following variables were derived for each sleep period:

1. Sleep onset (in hours:minutes): the time at which an athlete first fell asleep after going to bed;

2. Sleep offset (in hours:minutes): the time at which an athlete last woke before getting up;

3. Sleep duration (in hours): the amount of sleep obtained during a sleep period, that is, between sleep onset and sleep offset.

The athletes' sleep was monitored for an average of 12 (4) (mean [SD]) nights. Habitual values for the 3 objective sleep variables were calculated by averaging sleep onset, sleep offset, and sleep duration using the number of nights of data available for each athlete. In addition, a "sleep deficit index" was calculated for each athlete by subtracting "habitual sleep duration" (objective measure) from "sleep need" (subjective measure). 


\section{Statistical Analyses}

Descriptive analyses of athletes' subjective and objective sleep variables were conducted. All variables were normally distributed according to the Kolmogorov-Smirnov normality test.

The aims of the study were addressed by conducting a series of linear mixed effects models using the variance components covariance structure and restricted maximum likelihood estimation. To examine the difference between sleep need and habitual sleep duration for the entire sample, "sport" was entered as a random effect into the model and "type of measurement" (ie, sleep need or habitual sleep) was entered as a fixed effect. Separate models were then used to examine the difference between habitual sleep duration and sleep need in 9 of the 12 sports, with "type of measurement" entered as a fixed effect. (Please note: Alpine skiing $[n=1]$, diving $[n=1]$, and kayaking $[n=2]$ were not included in the sport-specific analyses.)

Two linear mixed effects models were used to examine the impact of habitual sleep onset time and habitual sleep offset time on habitual sleep duration. Habitual sleep duration was binned as a function of habitual sleep onset time $(9 \times 30$-min bins $)$ and habitual sleep offset time $(8 \times 30$-min bins). In each model, "bin" was entered as a fixed effect.

Three linear mixed effects models were used to examine differences in the 7 sleep variables $(3 \times$ subjective, $3 \times$ objective, $1 \times$ sleep deficit index) between individual and team sports, between all sports, and between the sexes. In each of the respective models, "sport type," "sport," and "sex" were entered as a single fixed effect.

Where appropriate, main effects were examined using pairwise comparisons with a Bonferroni adjustment. Within- and betweengroup effect sizes were calculated using Cohen $d$. Effect sizes were interpreted as follows: $0.2=$ small effect; $0.5=$ medium effect, and $0.8=$ large effect. All statistical analyses were performed using SPSS (version 26; IBM Corp, Armonk, NY). Results are reported as mean and SD and were considered significant at $P<.05$.

\section{Results}

\section{Habitual Sleep Duration, Sleep Need, and Sleep Deficit Index}

The participants had a habitual sleep duration of 6.7 (0.8) hours, which was significantly less than their self-assessed sleep need of 8.3 (0.9) hours $\left(F_{1,5.1}=211.03, P<.001 ; d=1.9\right.$, large $)$, resulting in a sleep deficit index of 96.0 (60.6) minutes (Table 2). Habitual sleep duration was significantly less than self-assessed sleep need for all sports (Figure 1).

The US National Sleep Foundation recommends that teenagers aged 14-17 years obtain 8 to 10 hours of sleep each night and young adults/adults aged 18-64 years obtain 7 to 9 hours of sleep each night. In this sample, $3 \%, 88 \%$, and $9 \%$ of participants had a self-assessed sleep need that was below, within, and above their age-specific range, respectively (Figure $2 \mathrm{~A}$ and $2 \mathrm{~B}$ ). Furthermore, 63\%, 37\%, and $0 \%$ of participants had a habitual sleep duration that was below, within, and above their age-specific range, respectively (Figure 2C and 2D). Only $3 \%$ of participants habitually obtained a sufficient amount of sleep to satisfy their self-assessed sleep need, and $71 \%$ of participants fell short by an hour or more (Figure $2 \mathrm{E}$ and $2 \mathrm{~F}$ ).

\section{Habitual Sleep Onset and Habitual Sleep Offset}

On average, participants had habitual sleep onset and habitual sleep offset times at 23:24 (00:42) and 07:18 (00:48) hours, respectively
(Table 2; Figures 3A and 4A). Habitual sleep duration was significantly affected by both habitual sleep onset time $\left(F_{8,165}=5.1\right.$, $P<.001 ; d=0.9-2.0$, large $)$ and habitual sleep offset time $\left(F_{9,165}=\right.$ $6.7, P<.001 ; d=1.0-3.2$, large); earlier onset times and later offset times both tended to result in greater habitual sleep duration (Figures 3B and 4B).

\section{Sleep Satisfaction and Sleep Quality}

Participants rated their sleep quality as 3.9 (0.9) on a Likert scale from 1 (very poor) to 6 (excellent) (Table 2). Similarly, participants rated their sleep satisfaction as 6.8 (1.6) on an arbitrary scale from 1 (very dissatisfied) to 10 (very satisfied) (Table 2).

\section{Sport-Based Comparisons}

There was a main effect of sport type (ie, individual vs team sport) on habitual sleep onset time, habitual sleep offset time, and habitual sleep duration (Figure 5A-5C). Habitual sleep onset and offset times were earlier in athletes from individual sport than athletes from team sports, but habitual sleep duration was shorter in athletes from individual sports than athletes from team sports. There was no main effect of sport type on sleep need $\left(F_{1,173}=1.40, P=.24\right.$; $d=0.2$, small $)$, sleep satisfaction $\left(F_{1,171}=0.15, P=.70 ; d=0.1\right.$, small $)$, sleep quality $\left(F_{1,171}=0.09, P=.76 ; d=0.1\right.$, small $)$, or sleep deficit index $\left(F_{1,173}=2.84, P=.09 ; d=0.3\right.$, small $)$.

There was a main effect of sport on habitual sleep onset time $\left(F_{10,160}=3.27, P=.001\right)$, habitual sleep offset time $\left(F_{10,160}=\right.$ $11.05, P<.001)$, and habitual sleep duration $\left(F_{10,160}=5.61\right.$, $P<.001)$ (Table 2). Habitual sleep onset was earliest in mountain bikers and latest in rugby union players (Figure 6A), habitual sleep offset was earliest in triathletes and latest in basketballers (Figure 6B), and habitual sleep duration was shortest in triathletes and longest in basketballers (Figure 6C). There was no main effect of sport on sleep need $\left(F_{10,160}=0.83, P=.60\right)$, sleep satisfaction $\left(F_{10,158}=0.86, P=.57\right)$, sleep quality $\left(F_{10,158}=1.53, P=.13\right)$, or sleep deficit index $\left(F_{10,160}=1.44, P=.17\right)$.

\section{Sex-Based Comparisons}

There was a main effect of sex on habitual sleep onset time (Figure 7A). Female athletes went to bed earlier than male athletes. There was no main effect of sex on habitual sleep offset time or habitual sleep duration (Figure 7B and 7C), nor was there an effect on sleep need $\left(F_{1,173}=0.17, P=.68 ; d=0.1\right.$, small $)$, sleep satisfaction $\left(F_{1,171}=0.06, P=.81 ; d=0.1\right.$, small $)$, sleep quality $\left(F_{1,171}=\right.$ $0.22, P=.64 ; d=0.1$, small $)$, or sleep deficit index $\left(F_{1,173}=2.15\right.$, $P=.15 ; d=0.3$, small) (Table 2).

\section{Discussion}

The primary findings of this study are (1) athletes need 8.3 hours of sleep to feel rested, (2) athletes typically obtain 6.7 hours of sleep, (3) the most sleep is obtained by athletes who fall asleep between 22:00 and 22:30 hours $(7.2 \mathrm{~h})$ or wake up between 09:00 and 09:30 hours $(7.6 \mathrm{~h}),(4)$ athletes involved in team sports $(6.9 \mathrm{~h})$ obtain more sleep than athletes involved in individual sports (6.4 h), and (5) female athletes have an earlier habitual sleep onset time than male athletes. Importantly, only $3 \%$ of athletes obtain enough sleep to satisfy their self-assessed sleep need, and $71 \%$ of athletes fall short by an hour or more. Insufficient or inadequate sleep, defined here as a failure to meet a required sleep need on a 


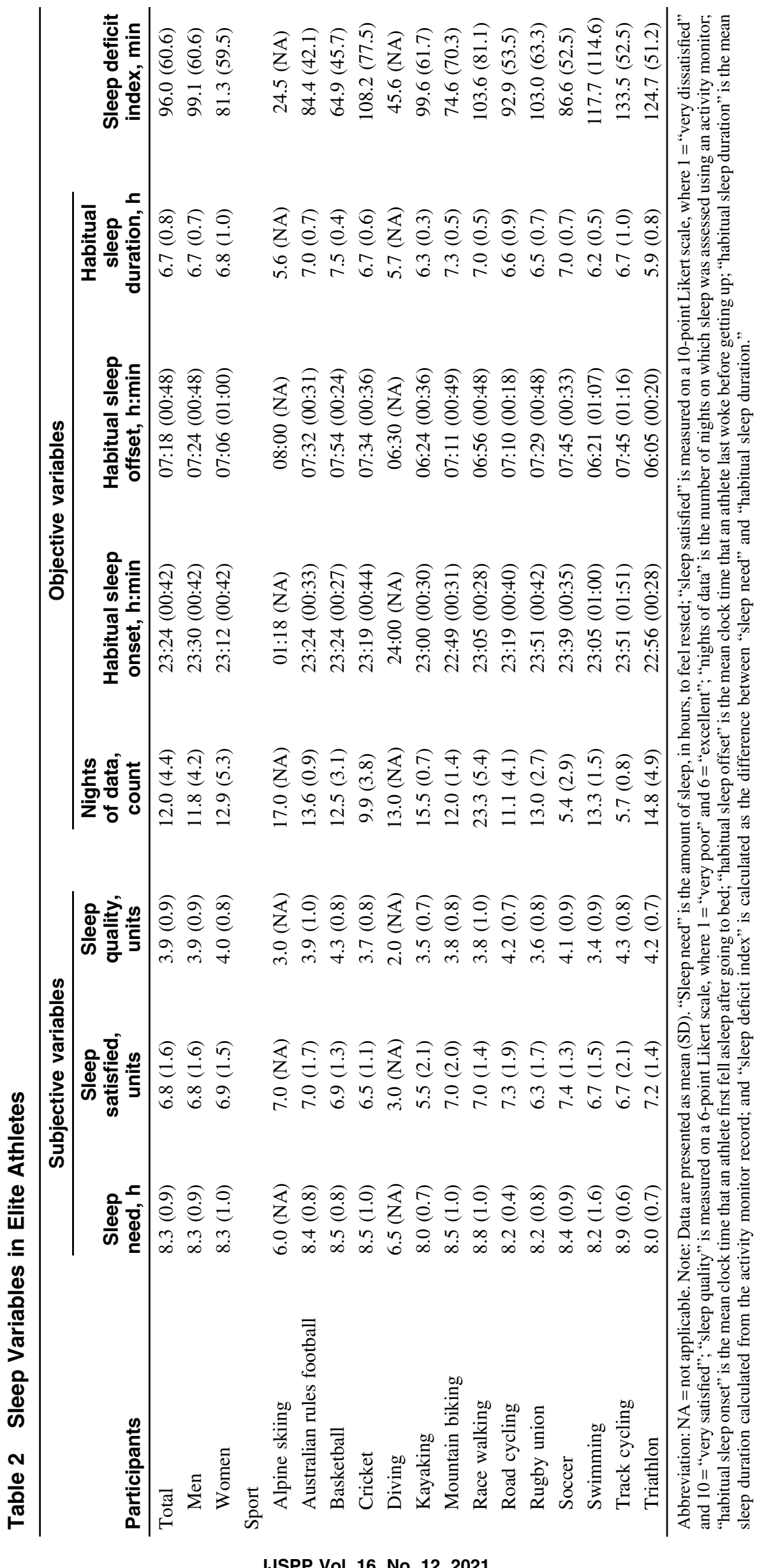




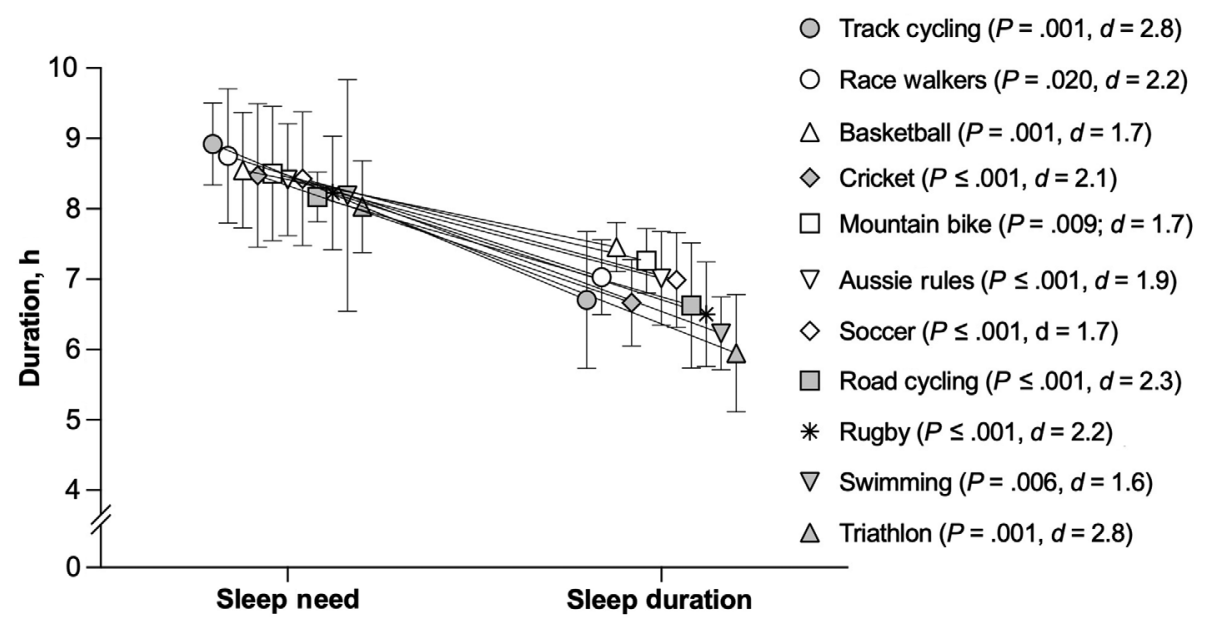

Figure 1 - Self-assessed sleep need compared with habitual sleep duration in athletes from 11 different sports. Mean values for each sport have been offset for interpretability. Error bars represent SD.
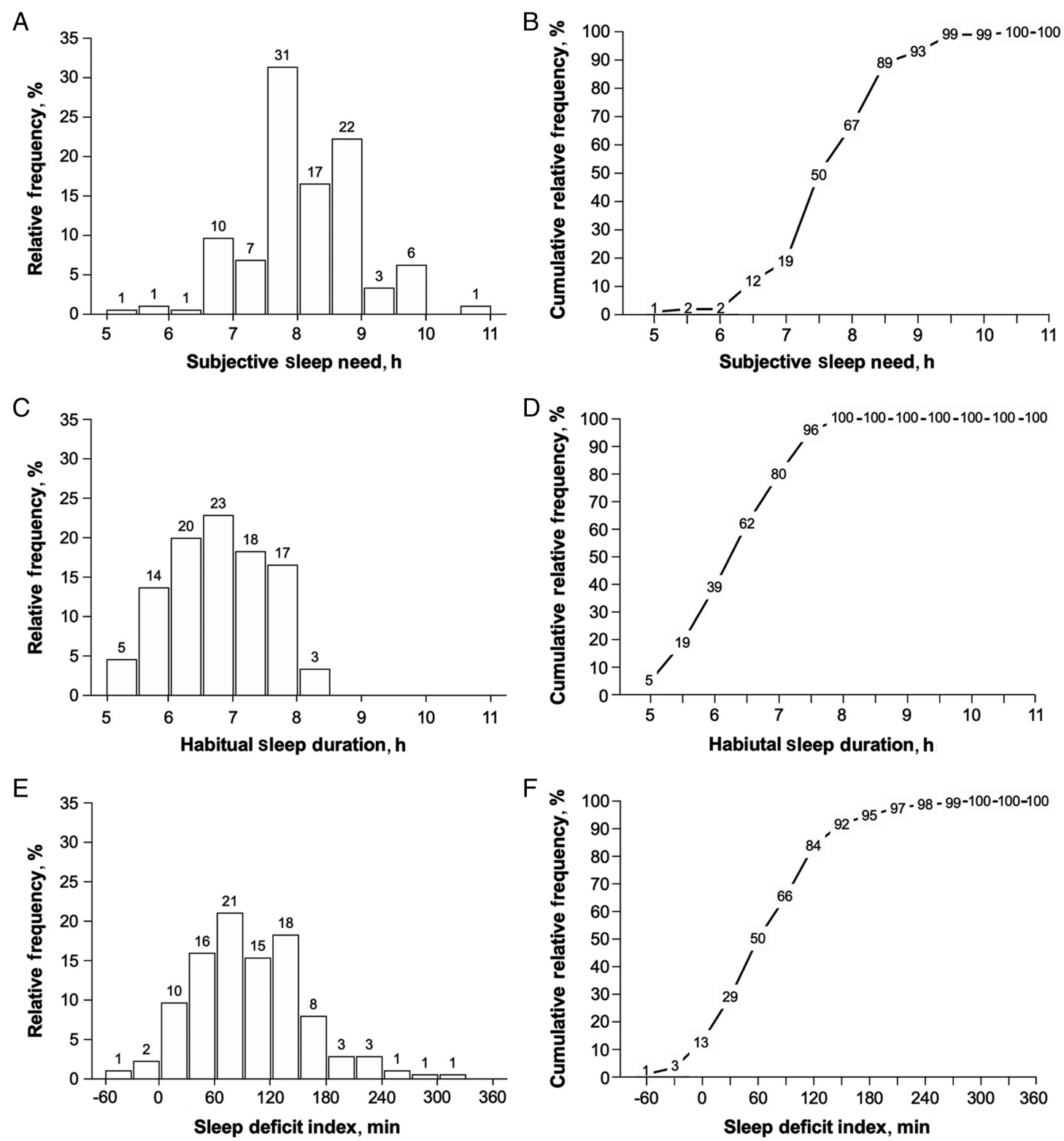

Figure 2 - Histograms representing the relative frequency and cumulative relative frequency of subjective sleep need (A and B), habitual sleep duration $(\mathrm{C}$ and $\mathrm{D})$, and sleep deficit index (E and $\mathrm{F})$. 

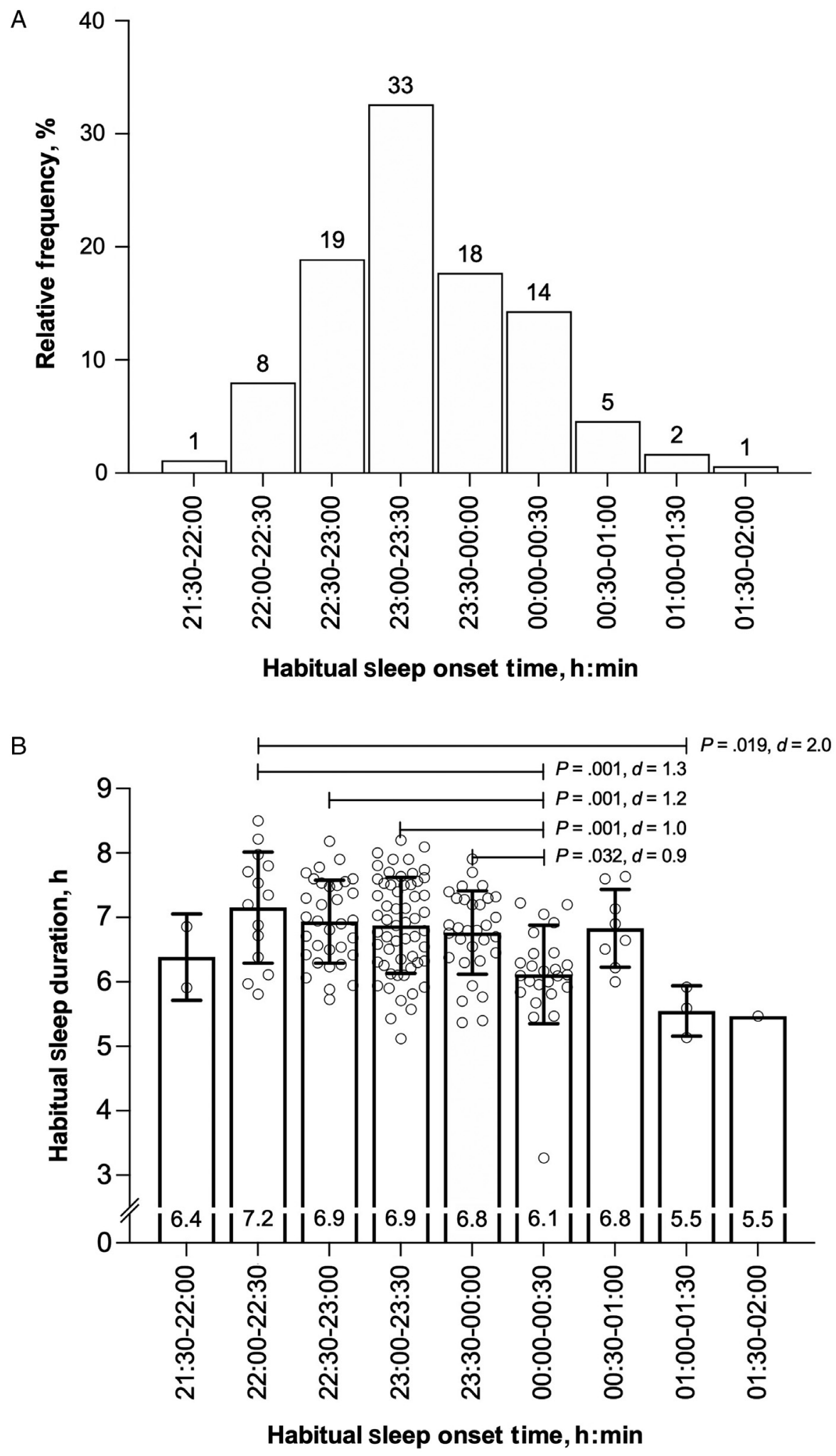

Figure 3 - Histogram representing the relative frequency of habitual sleep onset time (A) and bar charts (mean [SD]) with individual cases (open circles) representing habitual sleep duration plotted as a function of mean habitual sleep onset time (B). The outcomes of the post hoc comparisons between mean habitual sleep onset time bins and the corresponding effect sizes are presented in panel B. (Please note: The error bar on the final column in panel $\mathrm{B}$ is obscured because the value is small.) 


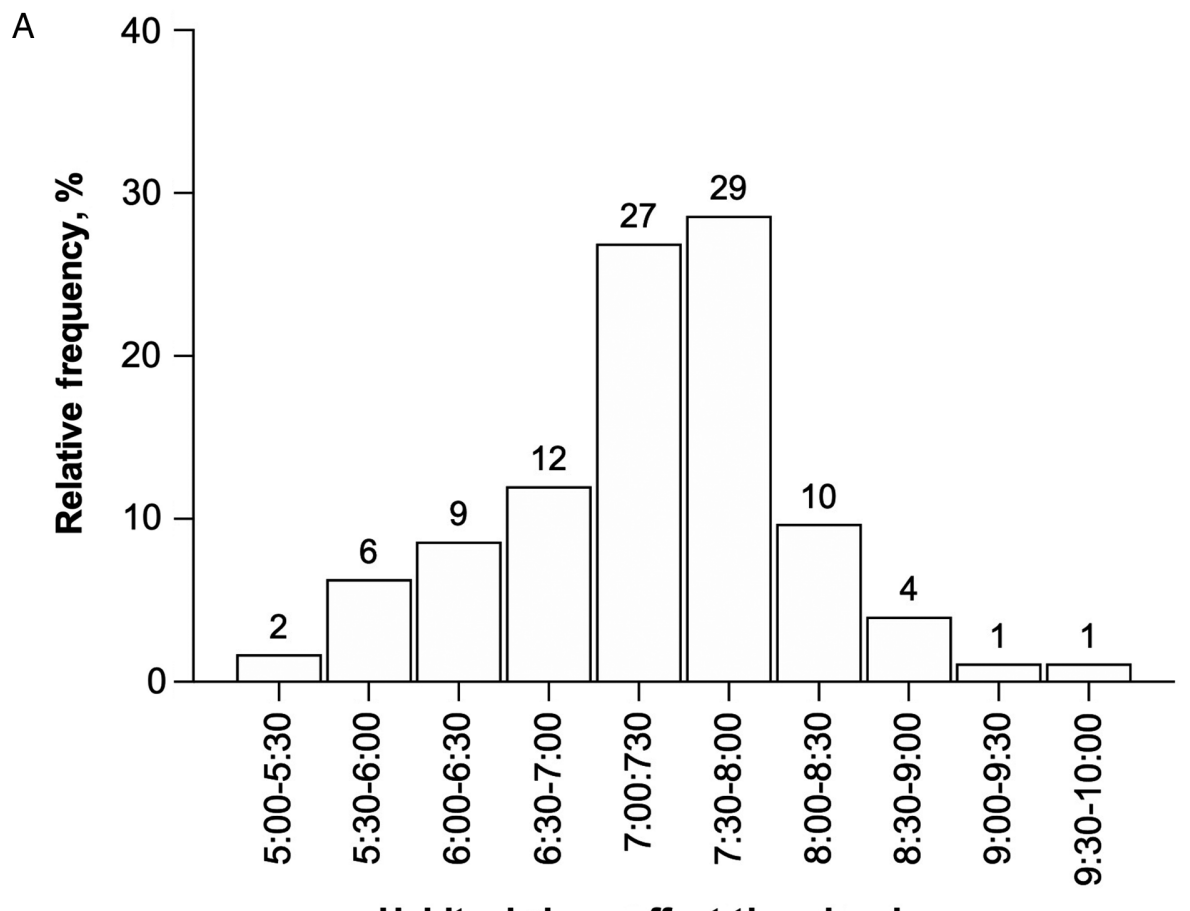

Habitual sleep offset time, h:min

B

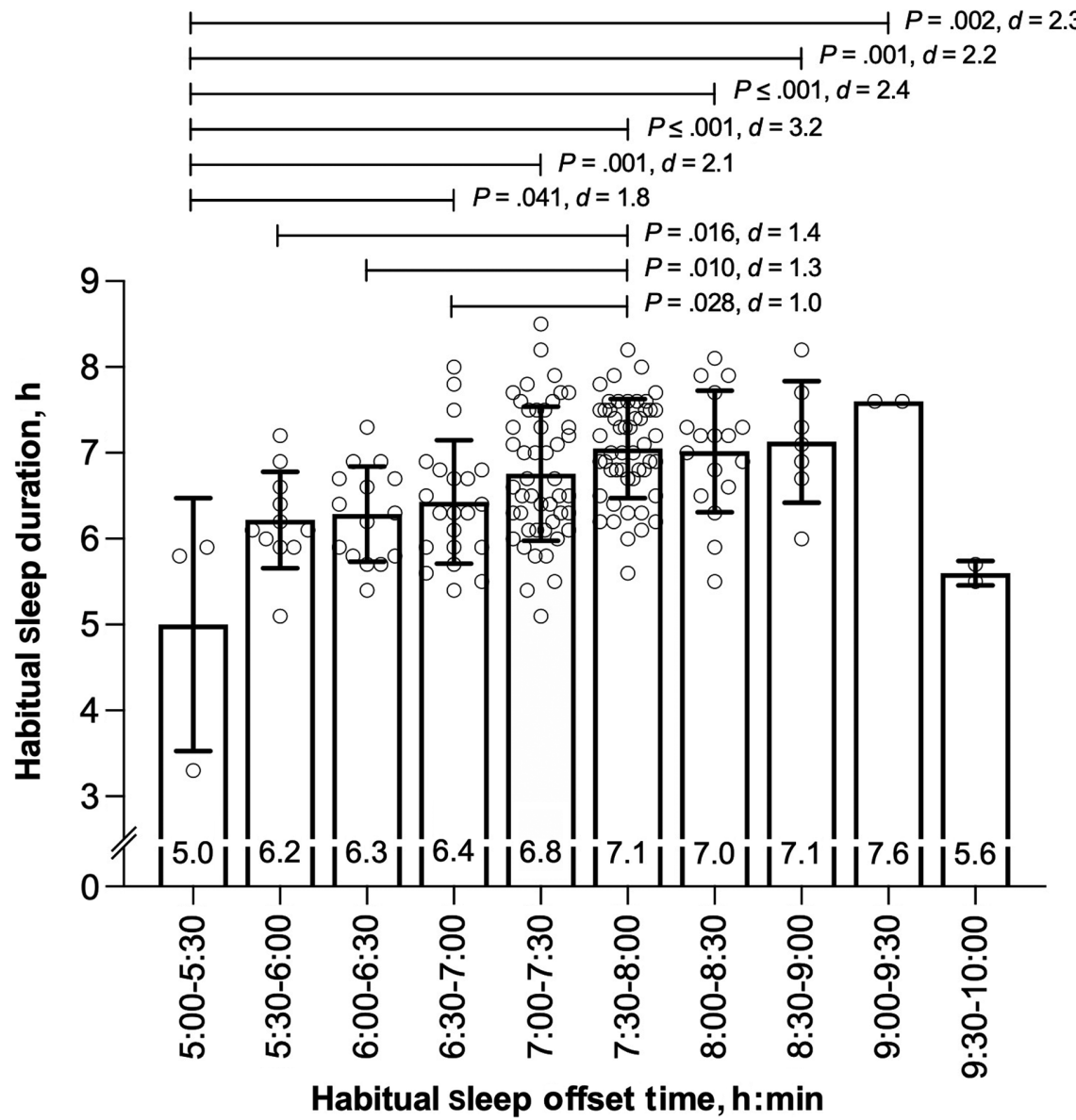

Figure 4 - Histogram representing the relative frequency of habitual sleep offset time (A) and bar charts (mean [SD]), with individual cases (open circles) representing habitual sleep duration plotted as a function of mean habitual sleep offset time (B). The outcomes of the post hoc comparisons between mean habitual sleep offset time bins and the corresponding effect sizes are presented in panel B. (Please note: The standard deviation for the penultimate column in panel $\mathrm{B}$ is equal to 0. .) 

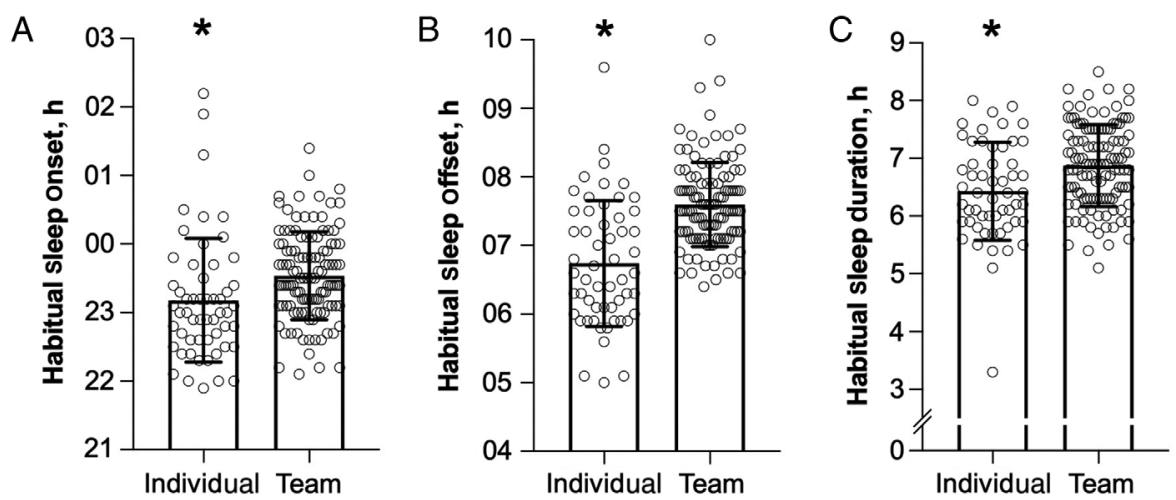

Figure 5 - Mean (SD) (bars and lines) and individual cases (open circles) of habitual sleep onset time (A), habitual sleep offset time (B), and habitual sleep duration (C) plotted as a function of individual sport or team sport. There was a main effect of sport type (ie, individual vs team sport, as indicated by $\left.{ }^{*}\right)$ on habitual sleep onset time $\left(F_{1,173}=9.15, P=.003 ; d=0.5\right.$, medium), habitual sleep offset time $\left(F_{1,173}=53.28, P<.001 ; d=1.2\right.$, large $)$, and habitual sleep duration $\left(F_{1,173}=13.1, P<.001 ; d=0.6\right.$, medium $)$.

regular basis, could have important consequences for an elite athlete, particularly in terms of their ability to train effectively and/or compete optimally.

The average subjective sleep need reported by elite athletes in this sample was 8.3 hours. Similar values have been reported by healthy untrained adolescents $(8.6 \mathrm{~h} ; 17$ [1] y) and adults $(8.0 \mathrm{~h} ; 36$ [12] y). ${ }^{23,24}$ Almost $80 \%$ of the current athletes reported needing between 7 and 9 hours of sleep, suggesting that the US National Sleep Foundation's sleep duration recommendation of 7 to 9 hours is reasonable for most athletes. However, sleep need varied between individual athletes, with the lowest reported sleep need of 5.5 hours $(n=1)$ and the highest reported sleep need of 11 hours $(n=2)$. A general recommendation may be appropriate for the majority of athletes, but the sleep need of some may substantially differ from the prescribed target.

In the present study, a sleep deficit index was calculated by subtracting athletes' habitual sleep duration from their subjective sleep need (ie, 96.0 [60.6] min). A difference of 1 hour between self-reported sleep need and sleep duration is typically considered insufficient sleep. ${ }^{25}$ In a large-scale epidemiological study with healthy, untrained adults $(n=12,423$; aged 33-60 y), the prevalence of insufficient sleep was $20 \%{ }^{25}$ In the present study, the prevalence of insufficient sleep was $71 \%$. There are 2 main alternatives that could explain a high prevalence of insufficient sleep in a population-either sleep need is higher than normal or the amount of sleep obtained is lower than normal. The latter explanation seems the one most likely to apply to the athletes in this study - the amount of sleep that they require is normal $(8.3 \mathrm{~h})$, but the amount of sleep they habitually obtain $(6.7 \mathrm{~h})$ is not sufficient to satisfy this requirement.

The average sleep duration observed in this cohort of elite athletes was 6.7 hours; however, this value varied between sports. In general, athletes from individual sports had earlier habitual sleep onset and offset times but obtained less sleep than athletes from team sports. Specifically, alpine skiing, diving, triathlon, swimming, kayaking, rugby union, and road cycling habitually obtained less sleep than the average, while athletes from basketball, mountain bike, race walking, Australian Rules Football, soccer, track cycling, and cricket habitually obtained more sleep than the average. For some sports, the habitual sleep durations observed in this study are similar to those reported previously (ie, Australian
Rules Football-7.0 h vs 6.9-7.1 h h $^{14,26,27}$; basketball-7.5 h vs $7.6 \mathrm{~h}^{7,28}$; soccer-7.0 h vs $\left.7.2 \mathrm{~h}\right),{ }^{29}$ but, for other sports, the current values are lower than those previously reported (ie, diving $-5.7 \mathrm{~h}$ vs $7.1 \mathrm{~h}^{12}$; rugby union-6.5 $\mathrm{h}$ vs $\left.7.1 \mathrm{~h}\right) .{ }^{30}$ It is not clear why the habitual sleep duration differs between current and past studies for some sports, but potential explanations include differences in the physical demands of training, ${ }^{31-33}$ the characteristics of the athletes (eg, chronotype), ${ }^{34}$ and/or the time of day that training sessions occur. ${ }^{8}$ It is plausible that longer habitual sleep durations could be achieved by manipulating aspects of an athlete's training schedule (especially for individual sports) to ensure bedtimes and getup times are optimized for sleep duration, but this is a question that is yet to be empirically investigated.

The amount of sleep an individual obtains on a regular basis does have implications for their ability to function effectively. A number of studies have examined the impact of severe, acute sleep loss on exercise and sports performance in athletes, that is, 1 to 2 nights of between 3 and 5 hours of time in bed, ${ }^{35}$ but there are no studies that have examined the impact of mild, chronic sleep loss on exercise and sports performance in elite athletes, that is, 7 to 14 days of between 5 and 7 hours of time in bed. In healthy, untrained adults, 7 days of either 5 or 7 hours of time in bed slows response time by $23 \%$ and $12 \%$, respectively, when compared with 9 hours of time in bed ${ }^{15}$; and 14 days of 6 hours of time in bed increases the rate of errors on a response time task by $177 \%$ when compared with 8 hours of time in bed. ${ }^{16}$ In the present study, $38 \%$ of athletes obtained 6.5 hours of sleep or less over an average of 12 (4) days. This level of habitual sleep duration could impair aspects of cognitive function and selfperceived capacity that are important for exercise and sports performance, for example, longer response times in time-critical sports, decreased time to fatigue in sports that require intermittent and repeated bouts of exercise, an increase in decision-making errors in any sport played over prolonged periods, and so forth. ${ }^{35}$ However, very little is known regarding the impact of short habitual sleep duration on exercise and sports performance. Short habitual sleep duration could directly affect exercise and sports performance through impairments in heart rate, minute ventilation, and lactate concentration, ${ }^{36}$ or it could indirectly affect exercise and sports performance through alterations in mood, motivation, and/or perceived exertion. ${ }^{37,38}$ In the absence of a 
A

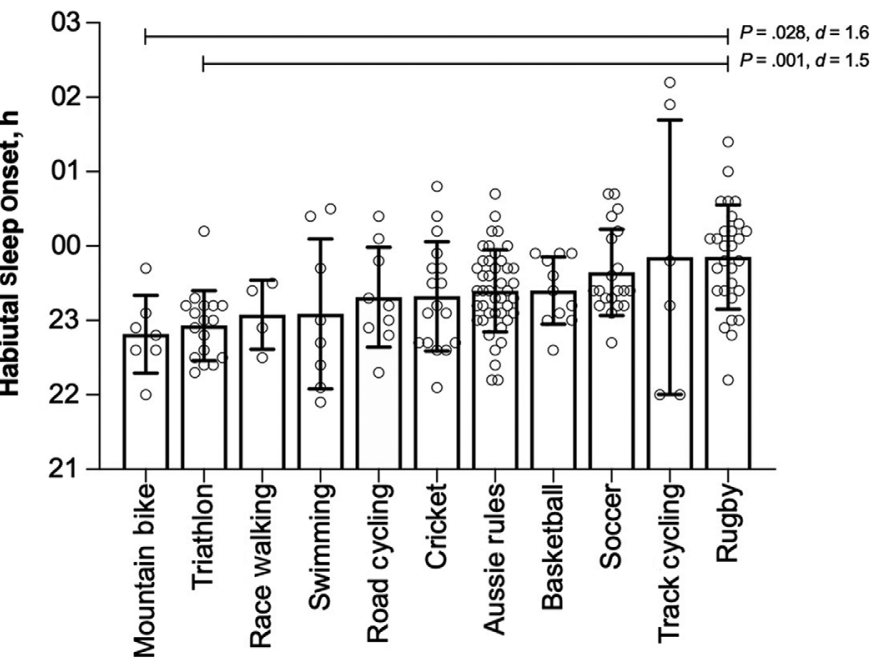

$\mathrm{B}$

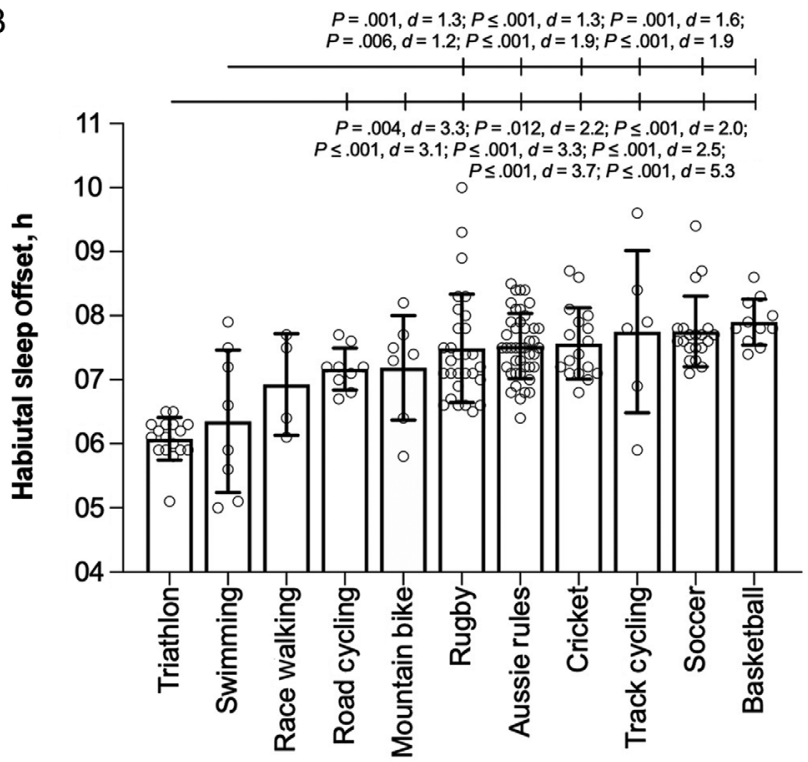

C

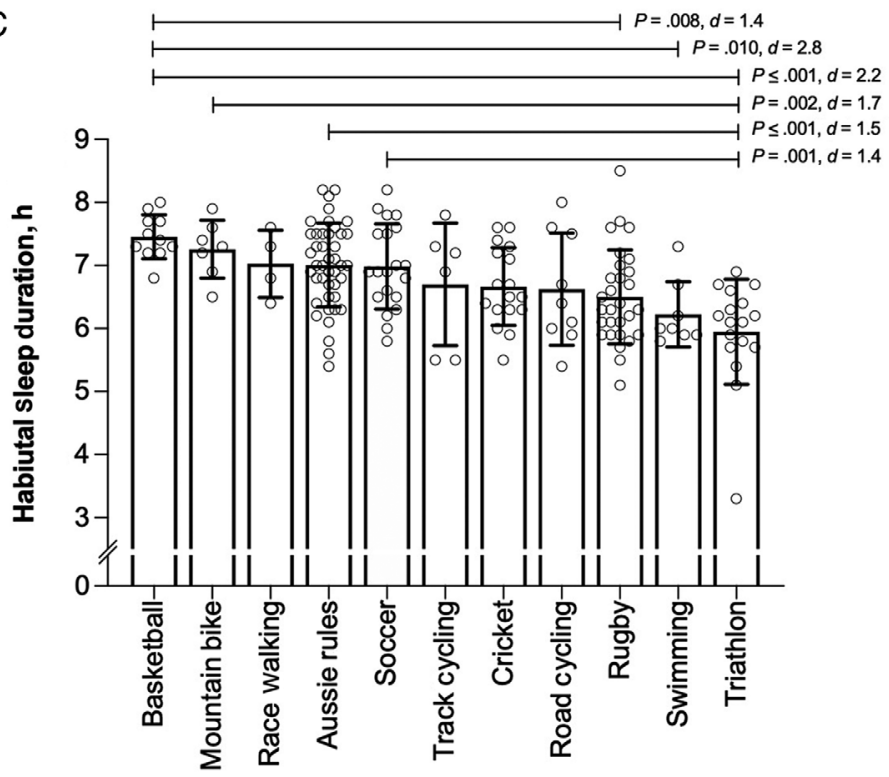

Figure 6 - Mean (SD) (bars and lines) with individual cases (open circles) representing habitual sleep onset (A), habitual sleep offset (B), and habitual sleep duration $(\mathrm{C})$ plotted for each sport. The outcomes of the post hoc comparisons between sports and the corresponding effect sizes are presented in each panel. In panel B, differences between swimming and the other sports are indicated by sequential vertical marks on the top line, and differences between triathlon and the other sports are indicated by sequential vertical marks on the bottom line. (Please note: The rank order in which sports are presented on the $x$-axis differs between the 3 panels.) 

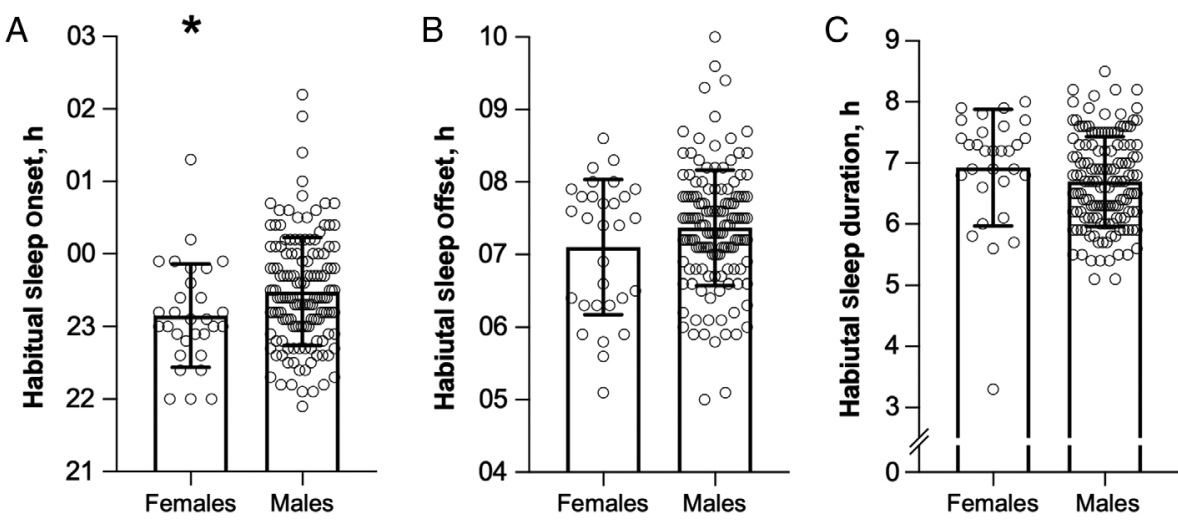

Figure 7 - Mean (SD) (bars and lines) and individual cases (open circles) of habitual sleep onset time (A), habitual sleep offset time (B), and habitual sleep duration $(\mathrm{C})$ for male and female athletes. Habitual sleep onset was earlier in females than males $\left(F_{1,173}=5.0, P=.03 ; d=0.5\right.$, medium, as indicated by $\left.{ }^{*}\right)$, but there was no difference between the sexes for habitual sleep offset time $\left(F_{1,173}=2.65, P=.11 ; d=0.3\right.$, small $)$ or habitual sleep duration $\left(F_{1,173}=\right.$ $2.15, P=.14 ; d=0.3$, small).

systematic evaluation of the relationship between habitual sleep duration and exercise and sports performance, it is not possible to confirm one explanation or the other.

Females are typically underrepresented in studies examining the sleep/wake behavior in athletes. Despite a large sample cohort in the present study $(\mathrm{n}=175)$, only $17 \%(\mathrm{n}=30)$ were women. Habitual sleep onset time was earlier in female athletes compared with male athletes, but there was no difference in habitual sleep duration between the sexes. Similarly, Leeder et $\mathrm{al}^{12}$ reported no difference in sleep duration between female $(n=43)$ and male $(n=23)$ Olympic athletes. However, this comparison-as was the comparison in the present studywas not sport specific. That is, male and female athletes from different sports were compared, rather than comparing sleep variables between male and female athletes from the same sport. Habitual sleep duration differs as a function of sport, ${ }^{6}$ which raises the possibility that potential sex differences in habitual sleep duration may be obscured if comparisons are not confined to athletes participating in the same sport. This is an area that requires further investigation.

There are some delimitations that should be considered when interpreting the results of the present study. Sleep need was assessed using a single subjective question. Objective sleep need can be assessed using sleep restriction and/or sleep extension protocols. ${ }^{39}$ However, these protocols are not feasible for use with elite athletes because they require an individual to spend multiple consecutive nights in a sleep laboratory. In the present study, habitual sleep duration was based on nighttime sleep episodes only-athletes were not required to record daytime naps. It is possible that some of the athletes supplemented their nighttime sleep with daytime naps. This would result in an underestimation of habitual sleep duration. Napping is an effective strategy in some situations when athletes' nighttime sleep is restricted ${ }^{40}$; however, the frequency of daytime napping in athletes is typically low ${ }^{6}$ and unlikely to substantially increase total sleep duration. ${ }^{9}$ Finally, activity monitors were used to assess habitual sleep duration. These devices are considered acceptable for monitoring sleep/wake behavior in the field, but validation studies indicate that the devices can either overestimate or underestimate sleep duration by 18 (52) and 54 (36) minutes, respectively. ${ }^{21,22}$ Consequently, the accuracy of the devices should be considered when interpreting the values of habitual sleep duration reported in the present study.

\section{Practical Applications}

The results presented here could be used by coaches and practitioners as normative data to guide their athletes regarding appropriate sleep targets for duration and timing. Importantly, elite athletes need $\sim 8$ hours of sleep per night to feel rested, but more than $70 \%$ of athletes do not obtain the sleep they need on a regular basis. Coaches and practitioners should consider factors that affect the timing of their athletes' sleep (eg, training start times, competition schedules, travel, etc), which may be preventing their athletes from obtaining the sleep they need. Potential strategies for maximizing sleep duration by manipulating sleep timing include (1) delaying the start time of morning training sessions and/or minimizing the number of training sessions that start before $6 \mathrm{AM}$ whenever possible; (2) encouraging athletes to delay their wake-up time the morning after an evening competition or training sessions, if practical; and (3) providing athletes with targets for sleep timing to help them achieve their optimal sleep duration where appropriate (eg, sleeps that start between 22:00 and 22:30 h or end between 09:00 and 09:30 h).

\section{Conclusions}

Elite male and female athletes need 8.3 hours of sleep to feel rested. However, a majority of athletes $(71 \%)$ fail to meet this need on most nights. The consequences of insufficient habitual sleep duration for general health and cognitive performance are well understood, but less is known regarding the impact of insufficient habitual sleep duration on exercise and sports performance. In the future, it will be important to determine whether increasing an athlete's habitual sleep is a feasible and efficacious strategy for improving exercise and sports performance.

\section{Acknowledgments}

This study was financially supported by the Australian Research Council under grant (LP0990371). The authors are grateful to the athletes and coaching staff for their time and commitment during data collection. 


\section{References}

1. Scharf MT, Naidoo N, Zimmerman JE, Pack A. The energy hypothesis of sleep revisited. Progress Neurobiol. 2008;86(3):264-280. doi:10.1016/j.pneurobio.2008.08.003

2. Vyazovskiy VV, Harris KD. Sleep and the single neuron: the role of global slow oscillations in individual cell rest. Nature Rev Neurosci. 2013;14(6):443-451. doi:10.1038/nrn3494

3. Krueger JM, Frank MG, Wisor JP, Roy S. Sleep function: toward elucidating an enigma. Sleep Med Rev. 2016;28:46-54. PubMed ID: 26447948 doi:10.1016/j.smrv.2015.08.005

4. Banks S, Van Dongen HPA, Maislin G, Dinges DF. Neurobehavioural dynamics following chronic sleep restriction: dose-response effects of one night for recovery. Sleep. 2010;33(8):1013-1026. PubMed ID: 20815182 doi:10.1093/sleep/33.8.1013

5. Hirshwkowitz M, Whiton K, Albert SM, et al. National Sleep Foundation's updated sleep duration recommendations: final report. Sleep Health. 2015;1(1):233-243. doi:10.1016/j.sleh.2015.10.004

6. Lastella M, Roach GD, Halson SL, Sargent C. Sleep/wake behaviours of elite athletes from individual and team sports. Eur J Sport Sci. 2015;15(2):94-100. PubMed ID: 24993935 doi:10.1080/17461391. 2014.932016

7. Lastella M, Roach GD, Vincent VE, Scanlan AT, Halson SL, Sargent C. The impact of training load on sleep during a 14-day training camp in elite, adolescent, female basketball players. Int J Sport Physiol Perf. 2020;15(5):724-730. doi:10.1123/ijspp.2019-0157

8. Sargent C, Lastella M, Halson SL, Roach GD. The impact of training schedules on the sleep and fatigue of elite athletes. Chronobiol Int. 2014; 31(10):1160-1168. PubMed ID: 25222347 doi:10.3109/07420528. 2014.957306

9. Sargent C, Halson S, Roach GD. Sleep or swim? Early-morning training severely restricts the amount of sleep obtained by elite swimmers. Eur J Sport Sci. 2014;14(suppl 1):S310-S315. PubMed ID: 24444223 doi:10.1080/17461391.2012.696711

10. Swinbourne R, Miller J, Smart D, Dulson DK, Gill N. The effects of sleep extension on sleep, performance, immunity and physical stress in rugby players. Sports. 2018;6(2):42. doi:10.3390/sports 6020042

11. Caia J, Thornton HR, Kelly VG, et al. Does self-perceived sleep reflect sleep estimated via activity monitors in professional rugby league athletes? J Sports Sci. 2017;36(13):1492-1496. PubMed ID: 29087784 doi:10.1080/02640414.2017.1398885

12. Leeder J, Glaister M, Pizzoferro K, Dawson J, Pedlar C. Sleep duration and quality in elite athletes measured using wristwatch actigraphy. J Sports Sci. 2012;30(6):541-545. PubMed ID: 22329779 doi:10.1080/02640414.2012.660188

13. O’Donnell S, Driller MW. Sleep-hygiene education improves sleep indices in elite female athletes. Int J Exerc Sci. 2017;10(4):522-530. PubMed ID: 28674597

14. Van Ryswyk E, Weeks R, Bandick L, et al. A novel sleep optimisation programme to improve athletes' well-being and performance. Eur J Sport Sci. 2017;17(2):144-151. PubMed ID: 27574901 doi:10. 1080/17461391.2016.1221470.

15. Belenky G, Wesensten NJ, Thorne DR, et al. Patterns of performance degradation and restoration during sleep restriction and subsequent recovery: a sleep dose-response study. J Sleep Res. 2003;12(1):1-12. PubMed ID: 12603781 doi:10.1046/j.1365-2869.2003.00337.x

16. Van Dongen HPA, Maislin G, Mullington JM, Dinges DF. The cumulative cost of additional wakefulness: dose-response effects on neurobehavioural functions and sleep physiology from chronic sleep restriction and total sleep deprivation. Sleep. 2003;26(2):117126. PubMed ID: 12683469 doi:10.1093/sleep/26.2.117
17. Van Dongen HPA, Maislin G, Dinges DF. Dealing with interindividual differences in the temporal dynamics of fatigue and performance: importance and techniques. Aviat Space Environ Med. 2004;75(3):A147-A154. PubMed ID: 15018277

18. Miller DJ, Sargent C, Vincent GE, Roach GD, Halson SL, Lastella M. Sleep/wake behaviours in elite athletes from three different football codes. J Sports Sci Med. 2017;16(4):604-605. PubMed ID: 29238263

19. Lastella M, Roach GD, Halson SL, Gore CJ, Garvican-Lewis LA, Sargent C. The effects of transmeridian travel and altitude on sleep: preparation for football competition. J Sports Sci Med. 2014;13(3): 718-720. PubMed ID: 25177205

20. Roach GD, Schmidt WF, Aughey RJ, et al. The sleep of elite athletes at sea level and high altitude: a comparison of sea-level natives and high-altitude natives (ISA3600). Brit J Sports Med. 2013;47(suppl 1): i114-i120. doi:10.1136/bjsports-2013-092843

21. Kosmadopoulos A, Sargent C, Darwent D, Zhou X, Roach GD. Alternatives to polysomnography (PSG): a validation of wrist actigraphy and a partial-PSG system. Behav Res Methods. 2014;46(4):1032-1041. PubMed ID: 24442593 doi:10.3758/s13428-013-0438-7

22. Sargent C, Lastella M, Halson SL, Roach GD. The validity of activity monitors for measuring sleep in elite athletes. J Sci Med Sport. 2016;19(10):848-853. PubMed ID: 26794719 doi:10.1016/j.jsams. 2015.12.007

23. Hysing M, Pallesen S, Stormark KM, Lundervold AJ, Sivertsen B. Sleep patterns and insomnia among adolescents: a population-based study. J Sleep Res. 2013;22(5):549-556. PubMed ID: 23611716 doi:10.1111/jsr.12055

24. Roepke SE, Duffy JF. Differential impact of chronotype on weekday and weekend sleep timing and duration. Nat Sci Sleep. 2010;2:213-220.

25. Hublin C, Kapiro J, Partinen M, Koskenvuo M. Insufficient sleep-a population based study in adults. Sleep. 2001;24(4):392-400. doi:10. 1093/sleep/24.4.392

26. Dennis J, Dawson B, Heasman J, Rogalski B, Robey E. Sleep patterns and injury occurrence in elite Australian footballers. J Sci Med Sport. 2016;19(2):113-116. PubMed ID: 25770421 doi:10.1016/j.jsams. 2015.02.003

27. Pitchford NW, Robertson SJ, Sargent C, Cordy J, Bishop DJ, Bartlett JD. Sleep quality but not quantity altered with a change in training environment in elite Australian Rules football players. Int J Sports Physiol Perform. 2017;12(1):75-80. PubMed ID: 27080592 doi:10. 1123/ijspp.2016-0009

28. Staunton C, Gordon B, Custovic E, Stanger J, Kingsley M. Sleep patterns and match performance in elite Australian basketball athletes. J Sci Med Sport. 2017;20(8):786-789. PubMed ID: 28169152 doi:10. 1016/j.jsams.2016.11.016

29. Robey E, Dawson B, Halson S, Gregson W, Goodman C, Eastwood P. Sleep quantity and quality in elite youth soccer players: a pilot study. Eur J Sport Sci. 2014;14(5):410-417. doi:10.1080/17461391. 2013.843024

30. Leduc C, Tee J, Phibbs P, et al. Objective sleep patterns and validity of self-reported sleep monitoring across different playing levels in rugby union. SA J Sports Med. 2020;32(1):1-6. doi:10.17159/2078516X/2020/v32i1a8456

31. Coutts AJ, Reaburn P. Monitoring changes in rugby league players' perceived stress and recovery during intensified training. Percep Motor Skill. 2008;106(3):904-916. doi:10.2466/pms.106.3.904-916

32. Killer SC, Svendsen IS, Jeukendrup AE, Gleeson M. Evidence of disturbed sleep and mood state in well-trained athletes during shortterm intensified training with and without a high carbohydrate nutritional intervention. J Sports Sci. 2017;35(14):1402-1410. PubMed ID: 26406911 doi:10.1080/02640414.2015.1085589 
33. Thornton HR, Duthie GM, Pitchford NW, Delaney JA, Benton DT, Dascombe BJ. Effects of a 2-week high-intensity training camp on sleep activity of professional rugby league athletes. Int J Sports Physiol Perform. 2017;12(7):928-933.

34. Lastella M, Roach GD, Halson SL, Sargent C. The chronotype of elite athletes. J Hum Kinet. 2016;54(1):219-225. PubMed ID: 28031772 doi:10.1515/hukin-2016-0049

35. Fullagar HHK, Skorski S, Duffield R, Hammes D, Coutts AJ, Meyer T. Sleep and athletic performance: the effects of sleep loss on exercise performance, and physiological and cognitive response to exercise. Sports Med. 2015;45(2):161-186. PubMed ID: 25315456 doi:10. 1007/s40279-014-0260-0

36. Mougin F, Bourdin H, Simon-Riguad ML, Didier JM, Toubin G, Kantelip P. Effects of a selective sleep deprivation on subsequent anaerobic performance. Int J Sports Med. 1996;17(02):115-119. PubMed ID: 8833713 doi:10.1055/s-2007-972818

37. Martin B. Effect of sleep deprivation on tolerance of prolonged exercise. Eur J Appl Physiol. 1981;47(4):345-354. doi:10.1007/BF02332962

38. Roberts SHS, Teo W-P, Aisbett B, Warmington SA. Extended sleep maintains endurance performance better than normal or restricted sleep. Med Sci Sports Exerc. 2019;51(12):2516-2523. PubMed ID: 31246714 doi:10.1249/MSS.0000000000002071

39. Ferrara M, De Gennaro L. How much sleep do we need? Sleep Med Rev. 2001;5(2):155-179. doi:10.1053/smrv.2000.0138

40. Romyn G, Lastella M, Miller DJ, Versey NG, Roach GD, Sargent C. Daytime naps can be used to supplement night-time sleep in athletes. Chronobiol Int. 2018;35(6):865-868. PubMed ID: 30024323 doi:10. 1080/07420528.2018.1466795 